\title{
ASYMPTOTICALLY PERIODIC SOLUTIONS OF A CLASS OF SECOND ORDER NONLINEAR DIFFERENTIAL EQUATIONS
}

\author{
LIANG ZHONGCHAO
}

ABSTRACT. In this paper we give necessary and sufficient conditions for all solutions of the system

$$
x^{\prime}=y, \quad y^{\prime}=-a(t) f(x) g(y)
$$

to be oscillatory or bounded, for all orbits of the system

$$
x^{\prime}=y, \quad y^{\prime}=-\alpha f(x) g(y)
$$

to be periodic, where $a(t) \rightarrow \alpha>0$ as $t \rightarrow \infty$, and for every orbit of (S) to approach a periodic orbit of $\left(\mathrm{S}_{1}\right)$. The conditions assuring that every solution of (S) is asymptotically periodic are also established.

1. Introduction. We consider the second order differential equation

$$
x^{\prime \prime}+a(t) f(x) g\left(x^{\prime}\right)=0,
$$

where $a: I \rightarrow R^{+}=(0, \infty), I=[\tau, \infty), f: R \rightarrow R=(-\infty, \infty)$ and $g: R \rightarrow R^{+}$are continuous functions, $a(t) \rightarrow \alpha>0$ as $t \rightarrow \infty$, and $x f(x)>0$ for $x \neq 0$. Assume that the solution of any Cauchy problem is unique and can be defined on $I$.

We also consider the limit equation of $(\mathrm{E})$,

$$
x^{\prime \prime}+\alpha f(x) g\left(x^{\prime}\right)=0,
$$

and the equivalent systems of $(E)$ and $\left(E_{1}\right)$,

$$
x^{\prime}=y, \quad y^{\prime}=-a(t) f(x) g(y)
$$

and

$$
x^{\prime}=y, \quad y^{\prime}=-\alpha f(x) g(y) .
$$

The purpose of the present paper is to establish necessary and sufficient conditions for all solutions of $(S)$ to be oscillatory or bounded, for all orbits of $\left(S_{1}\right)$ to be periodic, and for every orbit of $(S)$ to approach a periodic orbit of $\left(S_{1}\right)$ as $t \rightarrow \infty$, and also conditions to insure that every solution of $(\mathrm{S})$ is asymptotically periodic. Our results improve and extend some theorems in $[\mathbf{1}-\mathbf{8}]$.

Received by the editors June 21, 1985 and, in revised form, February 14, 1986.

1980 Mathematics Subject Classification (1985 Revision). Primary 34C25, 34C27.

Key words and phrases. Oscillation, periodic orbit, asymptotically periodic solution. 


\section{Equation (E).}

THEOREM 1. (E) is oscillatory if and only if

$$
\begin{array}{ll}
\mathrm{H}_{1}: & F(x)=\int_{0}^{x} f(u) d u \rightarrow \infty \quad \text { as }|x| \rightarrow \infty, \\
\mathrm{H}_{2}: & G(y)=\int_{0}^{y}(v / g(v)) d v \rightarrow \infty \quad \text { as }|y| \rightarrow \infty .
\end{array}
$$

ProOF. Sufficiency follows directly from [3, Theorem 1].

Necessity. If $F(\infty)<\infty$, then for given $x_{0}^{\prime}>0$, there exists $x_{0}>0$ such that

$$
F(\infty)-F\left(x_{0}\right)<\frac{1}{\gamma} G\left(x_{0}^{\prime}\right)
$$

where $\gamma=\sup _{t \geq \tau} a(t)$. By assumption on $a(t)$ clearly $0<\gamma<\infty$.

Let $x(t)$ be a solution of Cauchy problem (E) with $x(\tau)=x_{0}, x^{\prime}(\tau)=x_{0}^{\prime}$. By assumption, $x(t)$ and its derivative $x^{\prime}(t)$ are oscillatory. Let $T_{1}$ be the first zero of $x^{\prime}(t)$ on $I$. Clearly, $\tau<T_{1}<\infty$ and $x(t)>0$ for $t \in\left[\tau, T_{1}\right]$.

Integrating (E) from $\tau$ to $T_{1}$, we get

$$
\begin{aligned}
G\left(x^{\prime}\left(T_{1}\right)\right)-G\left(x^{\prime}(\tau)\right) & =-\int_{\tau}^{T_{1}} a(t) f(x(t)) x^{\prime}(t) d t \\
& \geq-\gamma\left[F\left(x\left(T_{1}\right)\right)-F(x(\tau))\right] .
\end{aligned}
$$

Then

$$
G\left(x_{0}^{\prime}\right) \leq \gamma\left(F(\infty)-F\left(x_{0}\right)\right)
$$

which contradicts (1). For the case $F(-\infty)<\infty$, the proof is similar, so $\mathrm{H}_{1}$ is necessary.

If $G(\infty)<\infty$, then for given $x_{0}<0$, there exists $x_{0}^{\prime}>0$ such that

$$
G(\infty)-G\left(x_{0}^{\prime}\right)<\beta F\left(x_{0}\right)
$$

where $\beta=\inf _{t \geq \tau} a(t)$. Clearly $0<\beta<\infty$.

Let $x(t)$ be a solution of Cauchy problem (E) with $x(\tau)=x_{0}, x^{\prime}(\tau)=x_{0}^{\prime}$. By assumption, $x(t)$ and $x^{\prime}(t)$ are oscillatory. Let $T_{2}$ be the first zero of $x(t)$ on $I$. Clearly $\tau<T_{2}<\infty$ and $x^{\prime}(t)>0$ for $t \in\left[\tau, T_{2}\right]$.

Integrating (E) from $\tau$ to $T_{2}$, we get

$$
\begin{aligned}
G\left(x^{\prime}\left(T_{2}\right)\right)-G\left(x^{\prime}(\tau)\right) & =-\int_{\tau}^{T_{2}} a(t) f(x(t)) x^{\prime}(t) d t \\
& \geq-\beta\left[F\left(x\left(T_{2}\right)\right)-F(x(\tau))\right] .
\end{aligned}
$$

Then

$$
G(\infty)-G\left(x_{0}^{\prime}\right) \geq \beta F\left(x_{0}\right)
$$

which contradicts (2). For the case $G(-\infty)<\infty$, the proof is similar, so $\mathrm{H}_{2}$ is necessary. This completes the proof.

REMARK 1. The sufficient condition of Theorem 1 extends [1, Theorem 0.1 and 5, Theorem 2].

Let $x(t)$ be a nontrivial oscillatory solution of (E). Clearly, $x(t)$ and $x^{\prime}(t)=y(t)$ are oscillatory and their zeros separate one another. Let $\left\{t_{2 n}\right\}$ and $\left\{t_{2 n+1}\right\}$ be 
sequences of zeros of $x(t)$ and $y(t)$ respectively such that $t_{2 n}<t_{2 n+1}<t_{2 n+2}$ $(n=0,1, \ldots), t_{n} \rightarrow \infty$ as $n \rightarrow \infty$.

Integrating (S) from $t_{2 k-1}$ to $t_{2 k}$, we obtain

$$
G\left(y\left(t_{2 k}\right)\right)=a\left(\tau_{2 k-1}\right) F\left(x\left(t_{2 k-1}\right)\right) \quad\left(t_{2 k-1}<\tau_{2 k-1}<t_{2 k}, k=1,2, \ldots\right) .
$$

Similarly, integrating (S) from $t_{2 k}$ to $t_{2 k+1}$, we obtain

$$
G\left(y\left(t_{2 k}\right)\right)=a\left(\tau_{2 k}\right) F\left(x\left(t_{2 k+1}\right)\right) \quad\left(t_{2 k}<\tau_{2 k}<t_{2 k+1}, k=0,1, \ldots\right) .
$$

Denote

$$
s_{n}=\prod_{k=1}^{n} \frac{a\left(\tau_{2 k-1}\right)}{a\left(\tau_{2 k}\right)}, \quad s_{n}^{\prime}=\prod_{k=1}^{n} \frac{a\left(\tau_{2 k-1}\right)}{a\left(\tau_{2 k-2}\right)} \quad(n=1,2, \ldots) .
$$

The sequences $\left\{s_{n}\right\}$ and $\left\{s_{n}^{\prime}\right\}$ are called characteristic sequences of the oscillatory solution $x(t)$. Clearly,

$$
s_{n}^{\prime}=\frac{a\left(\tau_{2 n}\right)}{a\left(\tau_{0}\right)} s_{n} \quad(n=1,2, \ldots)
$$

We have the following lemma.

LEMMA 1. Suppose that $x(t)$ is a nontrivial oscillatory solution of $(\mathrm{E})$. Then the sequences $\left\{x\left(t_{2 n+1}\right)\right\}$ and $\left\{x^{\prime}\left(t_{2 n}\right)\right\}$ satisfy (3) and (4), the sequences $\left\{s_{n}\right\}$ and $\left\{s_{n}^{\prime}\right\}$ satisfy (5).

LEMMA 2. Suppose that all solutions of (E) are bounded. Then (E) is oscillatory.

Lemma 2 is an immediate corollary of $[4$, Theorem 1$]$.

We need the following hypothesis:

$\mathrm{H}_{3}$ : (E) is oscillatory and the characteristic sequence $\left\{s_{n}\right\}$ of every nontrivial solution of $(\mathbf{E})$ is bounded.

THEOREM 2. All solutions of (E) and their derivatives are bounded if and only if $\mathrm{H}_{1}-\mathrm{H}_{3}$ hold.

PROOF. Sufficiency. By $\mathrm{H}_{1}, \mathrm{H}_{2}$, and Theorem 1, we know that any solution $x(t)$ of $(\mathrm{E})$ and $x^{\prime}(t)$ are oscillatory and hence by Lemma 1, (3)-(5) hold. From (3) and (4) we obtain

$$
F\left(x\left(t_{2 n+1}\right)\right)=s_{n} F\left(x\left(t_{1}\right)\right) \quad(n=1,2, \ldots)
$$

and

$$
G\left(x^{\prime}\left(t_{2 n}\right)\right)=s_{n}^{\prime} G\left(x^{\prime}\left(t_{0}\right)\right) \quad(n=1,2, \ldots) .
$$

From (6) and boundedness of the sequence $\left\{s_{n}\right\}$, we get

$$
F\left(x\left(t_{2 n+1}\right)\right) \leq s F\left(x\left(t_{1}\right)\right) \quad(n=1,2, \ldots),
$$

where $s_{n} \leq s<\infty$. From this estimate and $\mathrm{H}_{1}$ it follows that the sequence $\left\{x\left(t_{2 n+1}\right)\right\}$ is bounded, so that $x(t)$ is bounded.

From (5) and $\mathrm{H}_{3}$, we have

$$
s_{n}^{\prime} \leq \gamma s / a\left(\tau_{0}\right) \quad(n=1,2, \ldots)
$$


where $\gamma=\sup _{t \geq \tau} a(t)>0$, and in view of (7)

$$
G\left(x^{\prime}\left(t_{2 n}\right)\right) \leq \frac{\gamma s}{a\left(\tau_{0}\right)} G\left(x^{\prime}\left(t_{0}\right)\right) \quad(n=1,2, \ldots) .
$$

From this estimate and $\mathrm{H}_{2}$ it follows that the sequence $\left\{x^{\prime}\left(t_{2 n}\right)\right\}$ is bounded, so that $x^{\prime}(t)$ is bounded.

Necessity. By Lemma 2 (E) is oscillatory, and hence by Theorem $1 \mathrm{H}_{1}$ and $\mathrm{H}_{2}$ hold. Let $x(t)$ be any bounded oscillatory solution of $(\mathrm{E})$. From (6) we get

$$
s_{n}=F\left(x\left(t_{2 n+1}\right)\right) / F\left(x\left(t_{1}\right)\right) \leq L / F\left(x\left(t_{1}\right)\right) \quad(n=1,2, \ldots),
$$

where $L=\max _{|x| \leq M} F(x)$ and $M=\sup _{t \geq \tau}|x(t)|$. Thus $\left\{s_{n}\right\}$ is bounded. This complete's the proof.

COROLlARY 1. Suppose that the following holds.

$\mathrm{H}_{4}: a(t)$ is a function of bounded variation on $I$.

Then all solutions of $(\mathrm{E})$ and their derivatives are bounded if and only if $\mathrm{H}_{1}$ and $\mathrm{H}_{2}$ hold.

In fact, under the assumption on $a(t)$, it can be shown that every characteristic sequence $\left\{s_{n}\right\}$ is bounded, for which it is sufficient to show the convergence of the infinite product

$$
s_{\infty}=\prod_{k=1}^{\infty} \frac{a\left(\tau_{2 k-1}\right)}{a\left(\tau_{2 k}\right)}
$$

which is equivalent to the convergence of the series

$$
\sigma=\sum_{k=1}^{\infty} \frac{a\left(\tau_{2 k-1}\right)-a\left(\tau_{2 k}\right)}{a\left(\tau_{2 k}\right)}
$$

By $a(t) \rightarrow \alpha$ as $t \rightarrow \infty$ and $\mathrm{H}_{4}$, we have that

$$
\sum_{k=1}^{\infty}\left|\frac{a\left(\tau_{2 k-1}\right)-a\left(\tau_{2 k}\right)}{a\left(\tau_{2 k}\right)}\right|=\frac{1}{\beta} \sum_{k=1}^{\infty}\left|a\left(\tau_{2 k-1}\right)-a\left(\tau_{2 k}\right)\right|<\infty,
$$

where $\beta=\inf _{t \geq \tau} a(t)>0$. This implies that the series $\sigma$ is absolutely convergent and hence $s_{\infty}$ is convergent. So $\left\{s_{n}\right\}$ is bounded.

REMARK 2. The sufficiency condition of Corollary 1 improves and extends [2, Theorems 8 and 9; 7, Theorems 4 and 5; and 8, Theorems 1-3 and their corollary].

3. Equation $\left(\mathbf{E}_{1}\right)$. We note that there is one-to-one correspondence between periodic solutions of $\left(E_{1}\right)$ and periodic orbits of $\left(S_{1}\right)$, and that every orbit of $\left(S_{1}\right)$ is round the origin.

THEOREM 3. Every solution of $\left(\mathrm{E}_{1}\right)$ is periodic if and only if $\mathrm{H}_{1}$ and $\mathrm{H}_{2}$ hold.

Proof. Necessity. Since every periodic solution of $\left(\mathrm{E}_{1}\right)$ is oscillatory, by Theorem 1 we have $\mathrm{H}_{1}$ and $\mathrm{H}_{2}$.

Sufficiency. Consider the function $V(x, y)=\alpha F(x)+G(y)$. Taking the derivative of $V(x, y)$ along an orbit of $\left(\mathrm{S}_{1}\right)$ we get

$$
V^{\prime}(x, y)=\alpha f(x) y+(y / g(y))(-\alpha f(x) g(y)) \equiv 0 .
$$

Hence, from $\mathrm{H}_{1}$ and $\mathrm{H}_{2}$ it follows that $V(x, y)=C$ is a closed orbit of $\left(\mathrm{S}_{1}\right)$ for each $C>0$. This completes the proof.

REMARK 3. If $g(y) \equiv 1$, then [1, Theorem 0.1 and 5 , Theorem 2] can be derived from the sufficiency of Theorem 3 . 
4. Equations $(\mathbf{E})$ and $\left(\mathbf{E}_{\mathbf{1}}\right)$. In this and the next section we need the following hypothesis:

$\mathrm{H}_{5}$ : (E) is oscillatory and the characteristic sequence $\left\{s_{n}\right\}$ of every nontrivial solution of (E) satisfies $s_{n} \rightarrow s>0$ as $n \rightarrow \infty$.

THEOREM 4. Every orbit of $(\mathrm{S})$ approaches a periodic orbit of $\left(\mathrm{S}_{1}\right)$ in a spiral manner as $t \rightarrow \infty$ if and only if $\mathrm{H}_{1}, \mathrm{H}_{2}$, and $\mathrm{H}_{5}$ hold.

ProOF. Sufficiency. By $\mathrm{H}_{1}, \mathrm{H}_{2}$, and Theorem 3 every orbit of $\left(\mathrm{S}_{1}\right)$ is closed. By Theorems 1 and 2 we know that every solution $x(t)$ of $(\mathrm{E})$ and $x^{\prime}(t)=y(t)$ are oscillatory and bounded, and hence (6), (7), and (5) hold. Letting $n \rightarrow \infty$ in (6) and (7), by $\mathrm{H}_{5}$ and (5) we have

$$
\lim _{n \rightarrow \infty} F\left(x\left(t_{2 n+1}\right)\right)=s F\left(x\left(t_{1}\right)\right)
$$

and

$$
\lim _{n \rightarrow \infty} G\left(y\left(t_{2 n}\right)\right)=s^{\prime} G\left(y\left(t_{0}\right)\right)
$$

where $s^{\prime}=\alpha s / a\left(\tau_{0}\right)>0$. Letting $k \rightarrow \infty$ in (4), by (8) and (9) we find

$$
\alpha s F\left(x\left(t_{1}\right)\right)=s^{\prime} G\left(y\left(t_{0}\right)\right)=C_{1}>0 .
$$

Consider the periodic orbit of $\left(\mathrm{S}_{1}\right)$ :

$$
V(x, y)=\alpha F(x)+G(y)=C_{1} .
$$

Clearly, $V(x, y)=C_{1}$ intersects the $x$-axis at $\left(x_{1}, 0\right)$ and $\left(x_{2}, 0\right)$, and the $y$-axis at $\left(0, y_{1}\right)$ and $\left(0, y_{2}\right)$. Without loss of generality we assume that $x_{1}>0, x_{2}<0$, $y_{1}>0$, and $y_{2}<0$, and $x\left(t_{4 n-3}\right)>0, x\left(t_{4 n-1}\right)<0, y\left(t_{4 n-2}\right)>0$, and $y\left(t_{4 n}\right)<0$. From (8)-(10) it follows that $x\left(t_{4 n-3}\right) \rightarrow x_{1}, x\left(t_{4 n-1}\right) \rightarrow x_{2}, y\left(t_{4 n-2}\right) \rightarrow y_{1}$, and $y\left(t_{4 n}\right) \rightarrow y_{2}$ as $n \rightarrow \infty$. We now show that for arbitrary $\varepsilon>0$, there exists $\tau^{\prime} \geq \tau$ such that

$$
M(t)=(x(t), y(t)) \in A(\varepsilon)=\left\{(x, y): C_{1}-\varepsilon<V(x, y)<C_{1}+\varepsilon\right\}
$$

for all $t \geq \tau^{\prime}$. In fact, assume the contrary. Then by boundedness of $M(t)$, there exists a sequence $\left\{t_{j}^{\prime}\right\}, t_{j}^{\prime} \rightarrow \infty$ as $j \rightarrow \infty$, such that $M\left(t_{j}^{\prime}\right) \notin A(\varepsilon)$ and $M\left(t_{j}^{\prime}\right) \rightarrow$ $\bar{M}=(\bar{x}, \bar{y})$ as $j \rightarrow \infty$. Clearly, $\bar{M} \notin A(\varepsilon)$. Without loss of generality we assume $\bar{x}>0, \bar{y}>0$. Then $t_{4 n_{j}-3}<t_{j}^{\prime}<t_{4 n_{j}-2}$ for $j$ sufficiently large. Integrating (S) from $t_{4 n_{j}-3}$ to $t_{j}^{\prime}$ we obtain

$$
\begin{array}{r}
G\left(y\left(t_{j}^{\prime}\right)\right)+a\left(\tau_{4 n_{j}-3}^{\prime}\right) F\left(x\left(t_{j}^{\prime}\right)\right)=a\left(\tau_{4 n_{j}-3}^{\prime}\right) F\left(x\left(t_{4 n_{j}-3}\right)\right) \\
\left(t_{4 n_{j}-3}<\tau_{4 n_{j}-3}^{\prime}<t_{j}^{\prime}\right) .
\end{array}
$$

Letting $j \rightarrow \infty$ in (11), we have $V(\bar{x}, \bar{y})=C_{1}$, which is impossible.

Necessity. By assumption we know that any solution $(x(t), y(t))$ of $(\mathrm{S})$ is oscillatory and bounded, and hence by Theorem $2 \mathrm{H}_{1}$ and $\mathrm{H}_{2}$ hold.

As before, we assume $x\left(t_{4 n-3}\right) \rightarrow x_{1}>0$ and $x\left(t_{4 n-1}\right) \rightarrow x_{2}<0$ as $n \rightarrow \infty$, and that $V\left(x_{1}, 0\right)=V\left(x_{2}, 0\right)=C_{1}>0$, from which we have $F\left(x_{1}\right)=F\left(x_{2}\right)=C_{1} / \alpha>$ 0 . On the other hand, from (6) it follows that

$$
s_{2 k-2}=F\left(x\left(t_{4 k-3}\right)\right) / F\left(x\left(t_{1}\right)\right),
$$


and

$$
s_{2 k-1}=F\left(x\left(t_{4 k-1}\right)\right) / F\left(x\left(t_{1}\right)\right) \text {. }
$$

Letting $k \rightarrow \infty$ in (12) and (13), we obtain

$$
\lim _{k \rightarrow \infty} s_{2 k-2}=F\left(x_{1}\right) / F\left(x\left(t_{1}\right)\right)=C_{1} /\left[\alpha F\left(x\left(t_{1}\right)\right)\right]
$$

and

$$
\lim _{k \rightarrow \infty} s_{2 k-1}=F\left(x_{2}\right) / F\left(x\left(t_{1}\right)\right)=C_{1} /\left[\alpha F\left(x\left(t_{1}\right)\right)\right]
$$

Then we have

$$
\lim _{n \rightarrow \infty} s_{n}=C_{1} /\left[\alpha F\left(x\left(t_{1}\right)\right)\right]>0 .
$$

This completes the proof.

COROLlARY 2. Suppose that $\mathrm{H}_{4}$ holds. Then every orbit of (S) approaches a periodic orbit of $\left(\mathrm{S}_{1}\right)$ in a spiral manner as $t \rightarrow \infty$ if and only if $\mathrm{H}_{1}$ and $\mathrm{H}_{2}$ hold.

In fact, as in the proof of Corollary 1 , the infinite product $s_{\infty}$ is convergent. From this and $a(t)>0$ for $t \in I$ it follows that $s_{\infty}>0$. That is equivalent to $s_{n} \rightarrow s_{\infty}$ as $n \rightarrow \infty$ so that Corollary 2 can be derived from Theorem 4 .

REMARK 4. The sufficiency condition of Corollary 2 improves [6, Theorems 5 and 6].

5. Asymptotically periodic solutions. A solution $(x(t), y(t))$ of $(\mathrm{S})$ is defined to be asymptotically periodic if there exists a constant $T>0$ such that for arbitrary $\varepsilon>0$, there is a $\tau^{\prime} \geq \tau$ so that

$$
|(x(t+T), y(t+T))-(x(t), y(t))|<\varepsilon \quad \text { for all } t \geq \tau^{\prime}
$$

(see $[6, \S 5])$.

THEOREM 5. Suppose that $\mathrm{H}_{1}, \mathrm{H}_{2}$, and $\mathrm{H}_{5}$ hold. Then every solution $(x(t), y(t))$ of $(\mathrm{S})$ is asymptotically periodic.

PROOF. As in the proof of Theorem 4, we assume that $x\left(t_{4 n-3}\right) \rightarrow x_{1}>0$, $x\left(t_{4 n-1}\right) \rightarrow x_{2}<0, y\left(t_{4 n-2}\right) \rightarrow y_{1}>0$, and $y\left(t_{4 n}\right) \rightarrow y_{2}<0$ as $n \rightarrow \infty$. Clearly, $\left(x\left(t+t_{4 n-3}\right), y\left(t+t_{4 n-3}\right)\right)$ is a solution of the Cauchy problem

$$
u^{\prime}=v, v^{\prime}=-\alpha f(u) g(v)+\left(\alpha-a\left(t+t_{4 n-3}\right)\right) f\left(x\left(t+t_{4 n-3}\right)\right) g\left(y\left(t+t_{4 n-3}\right)\right)
$$

with $u(0)=x\left(t_{4 n-3}\right), v(0)=0$. Let $\left(x^{*}(t), y^{*}(t)\right)$ be a solution of the Cauchy problem $\left(\mathrm{S}_{1}\right)$ with $x^{*}(0)=x_{1}, y^{*}(0)=0$. By Theorem $3,\left(x^{*}(t), y^{*}(t)\right)$ is a periodic solution of $\left(\mathrm{S}_{1}\right)$ (periodic $\left.T>0\right)$. Using Yoshizawa's method (see $[9, \S 13]$ ), it is easy to prove that $\left(x\left(t+t_{4 n-3}\right), y\left(t+t_{4 n-3}\right)\right)$ converges to $\left(x^{*}(t), y^{*}(t)\right)$ uniformly in $t \in[0,3 T]$ as $n \rightarrow \infty$. Hence, for $\varepsilon>0$, there exists an integer $N>0$ such that $t_{4(n+1)-3}-t_{4 n-3}<2 T$ and $\left|\left(x\left(t+t_{4 n-3}\right), y\left(t+t_{4 n-3}\right)\right)-\left(x^{*}(t), y^{*}(t)\right)\right|<\varepsilon / 2$ for $t \in[0,3 T]$ and $n \geq N$. From this we have

$$
\begin{aligned}
\mid(x(t+ & \left.\left.T+t_{4 n-3}\right), y\left(t+T+t_{4 n-3}\right)\right)-\left(x\left(t+t_{4 n-3}\right), y\left(t+t_{4 n-3}\right)\right) \mid \\
\leq & \left|\left(x\left(t+T+t_{4 n-3}\right), y\left(t+T+t_{4 n-3}\right)\right)-\left(x^{*}(t+T), y^{*}(t+T)\right)\right| \\
& +\left|\left(x^{*}(t), y^{*}(t)\right)-\left(x\left(t+t_{4 n-3}\right), y\left(t+t_{4 n-3}\right)\right)\right| \\
& <\varepsilon \quad \text { for } t \in[0,2 T] .
\end{aligned}
$$


Furthermore, we obtain

$$
|(x(t+T), y(t+T))-(x(t), y(t))|<\varepsilon \quad \text { for all } t \geq t_{4 N-3} .
$$

This completes the proof.

COROLlaRY 3. Suppose that $\mathrm{H}_{1}, \mathrm{H}_{2}$, and $\mathrm{H}_{4}$ hold. Then the conclusion of Theorem 5 holds.

REMARK 5. Corollary 3 improves [6, Theorem 7 and its Corollary].

\section{REFERENCES}

1. N. P. Bhatia, Some oscillation theorems for second order differential equations, J. Math. Anal. Appl. 15 (1966), 442-446.

2. I. Bihari, Researchers of the boundedness and stability of the solutions of nonlinear differential equations, Acta Math. Hungar. 8 (1957), 261-278.

3. Liang Zhongchao, Asymptotic character of the solutions of a class of second order nonlinear differential equations, Shuxue Jinzhan, 9 (1966), 251-264. MR 38 \#4767.

4. Liang Zhongchao and Chen Shaozhu, The asymptotic behavior of solutions to second order nonlinear differential equations, Chinese Ann. Math. Ser. B 6 (1985), 481-490.

5. W. R. Utz, Properties of solutions of $u^{\prime \prime}+g(t) u^{2 n-1}=0$, Monatsh. Math. 66 (1962), $55-60$.

6. J. S. W. Wong and T. A. Burton, Some properties of solutions of $u^{\prime \prime}+a(t) f(u) g\left(u^{\prime}\right)=0$. (II), Monatsh. Math. 69 (1965), 368-374.

7. J. S. Wong, Some properties of solutions of $u^{\prime \prime}+a(t) f(u) g\left(u^{\prime}\right)=0$. (III), SIAM J. Appl. Math. 14 (1966), 209-214.

8. $\ldots$, Boundedness theorems of solutions of $u^{\prime \prime}+a(t) f(u) g\left(u^{\prime}\right)=0$. (IV), Enseign. Math. 13 (1967), 157-165.

9. T. Yoshizawa, Stability theory by Liapunov's second method, Math. Soc. Japan, Tokyo, 1966.

Department of Mathematics, Shandong College of Oceanography, QINGDaO, Shandong, The People's Republic of China 